\title{
Cultiver ses relations
}

\author{
par Douglas Sellinger
}

$\mathrm{E}^{\mathrm{n}}$

tant que parent, j'aime cultiver ma relation avec ma fille

alors même qu'elle sort de l'adolescence et devient une jeune femme extraordinaire. Elle a comme objectif d'entamer prochainement des études en pharmacie et j'ai bien hâte que ma fille devienne aussi ma collègue. Comme c'est le cas dans toutes les familles, notre relation évolue sans cesse, parfois en douceur et parfois plus difficilement.

La relation entre la Société canadienne des pharmaciens d'hôpitaux (SCPH) et l'Association des pharmaciens du Canada (APhC), deux des principales organisations de valorisation des pharmaciens au Canada, évolue elle aussi continuellement. Elle se caractérise parfois par des difficultés alors qu’à certains moments il en émane une forte collaboration.

Le nouveau règlement administratif de la $\mathrm{SCPH}$, adopté au cours de l'assemblée générale annuelle de la Société en août 2013, est entré en vigueur le 1er mai 2014. La Loi canadienne sur les organisations à but non lucratif et les règlements afférents ont modifié la façon dont ces organisations sont structurées et comment elles peuvent agir. Ces modifications apportées aux règlements touchent aussi bon nombre de nos partenaires stratégiques externes, dont l'APhC. Celle-ci profite de son propre examen de conformité pour faire peau neuve. Elle a présenté la réforme de son modèle d'adhésion et de gouvernance ainsi que de la structure de son conseil à ses membres au cours de son assemblée générale annuelle en 2013. Ces changements auront une incidence sur les relations qu'entretient l'APhC avec ses partenaires externes, notamment la SCPH.

En août dernier, le bureau de la SCPH a invité la présidente et le chef de la direction de l'APhC à participer à la Journée des enjeux du conseil national afin quils présentent leur nouveau modèle d'adhésion. L'APhC passera ainsi d'un modèle où les membres sont des personnes à un modèle où seules des organisations sont membres. Seules les associations nationales et provinciales de pharmacie pourront ainsi être membres de l'APhC. Les personnes membres de ces associations obtiendront les avantages offerts par l'APhC par l'intermédiaire de leur affiliation à l'une de ces organisations. L'APhC a demandé à

Douglas Sellinger, BSP, MALT, est président sortant et agent de liaison

externe pour la Société canadienne des pharmaciens d'hôpitaux.

la SCPH de devenir l'une de ses organisations membres. Le Conseil ne s'est pas encore engagé dans cette voie. Il cherche toujours à mieux connaitre les avantages que pourraient retirer la $\mathrm{SCPH}$ d'une adhésion à l'APhC ainsi que les coûts qui y sont rattachés afin de déterminer si les membres de la $\mathrm{SCPH}$ la considéreront comme avantageuse dans son ensemble.

À titre d'organisations nationales valorisant les pharmaciens et la pharmacie, la $\mathrm{SCPH}$ et l'APhC ont travaillé ensemble sur bon nombre de projets, notamment sur le programme ADAPT, le Plan directeur pour la pharmacie, le Réseau de spécialistes en pharmacie en soins de santé primaires et, plus récemment, différents projets portant sur les pénuries de médicaments. La SCPH et l'APhC ont œuvré de concert avec d'autres partenaires stratégiques à la création de la base de données canadienne des pénuries de médicaments (www.penuriesdemedicaments.ca) et au sein du Comité directeur multilatéral sur les pénuries de médicaments au Canada (qui a approuvé le Protocole pour la divulgation et la communication des pénuries de médicaments et Une boîte à outils pour mieux comprendre les interventions aux pénuries de médicaments au Canada et en accroître la transparence). De plus, la SCPH et l'APhC se sont associées afin de mener des sondages qui visaient à connaître les répercussions des pénuries de médicaments sur les patients et les pharmaciens et de diffuser cette information.

Malgré les changements au sein de l'APhC et que la SCPH décide ou non de devenir une organisation membre de l'APhC, nous continuerons à compter cette association parmi nos principaux partenaires stratégiques et nous envisageons avec plaisir toutes futures collaborations avec elle chaque fois que les enjeux des pharmaciens concorderont entre nos deux grandes organisations. 